\title{
On dispersive representation of kaon and eta decays to 3 pions
}

\author{
Martin Zdráhal, ${ }^{a}{ }^{*}{ }^{*}$ Karol Kampf, ${ }^{a}$ Marc Knecht ${ }^{b}$ and Jiří Novotný ${ }^{a}$ \\ ${ }^{a}$ Institute of Particle and Nuclear Physics, Faculty of Mathematics and Physics, Charles University, \\ V Holešovičkách 2, CZ-180 00 Prague 8, Czech Republic \\ ${ }^{b}$ Centre de Physique Théorique, CNRS/Aix-Marseille Univ./Univ. du Sud Toulon-Var(UMR 7332) \\ CNRS-Luminy Case 907, 13288 Marseille Cedex 9, France \\ E-mail: zdrahal@ipnp.mff.cuni.cz, kampf@ipnp.mff.cuni.cz, \\ Marc.Knecht@cpt.univ-mrs.fr, novotny@ipnp.mff.cuni.cz
}

We present and develop a general dispersive framework allowing us to construct representations of the amplitudes for the processes $P \pi \rightarrow \pi \pi, P=K, \eta$, valid at the two-loop level in the low-energy expansion. The construction proceeds through a two-step iteration, starting from the tree-level amplitudes and their S and P partial-wave projections. The one-loop amplitudes are obtained for all possible configurations of pion masses. The second iteration is performed in the cases where either all masses of charged and neutral pions are equal or for the decay into three neutral pions. Issues related to analyticity properties of the amplitudes and their lowest partial-wave projections are given particular attention.

40th International Conference on High Energy physics - ICHEP2020

July 28 - August 6, 2020

Prague, Czech Republic (virtual meeting)

\footnotetext{
${ }^{*}$ Speaker
} 


\section{Introduction}

This contribution is partially based on [1], where a more detailed discussion of the technical aspects and also a more complete list of references can be found.

During the last decades, the experimental knowledge of the $K^{ \pm}, K_{L}$ and $\eta$ decays to three pions has greatly improved. We have high statistics data for them (see, e.g., [2-5]). In all the relevant cases, there exist observations and also analyses of the cusp effect. There are also other measurements and analyses on-going or planned $[6,7]$.

These decays live in the realm of low-energy quantum chromodynamics (QCD), so the applicable theories are chiral perturbation theory, lattice QCD and similar. These processes are important for the studies of QCD in this regime. The studies of decays of $\eta$ lead to the determination of the $m_{u}-m_{d}$ quark masses (see, e.g. [8]) and can give limits on CP violations [9]. Those processes with the cusp effect can be used for the determination of the $\pi \pi$ scattering lengths [10].

\section{Model-independent description of the data}

Traditionally, for comparing experimental data with theories, the Dalitz plot parametrization has been used (see, e.g. [11]). For instance, for $\eta(k) \rightarrow \pi_{1}\left(p_{1}\right) \pi_{2}\left(p_{2}\right) \pi_{3}\left(p_{3}\right)$, with Mandelstam variables $s_{i}=\left(k-p_{i}\right)^{2}$, one introduces kinematic variables

$$
x=\frac{\sqrt{3}}{2 m_{\eta} Q}\left(s_{2}-s_{1}\right), \quad y=\frac{3}{2 m_{\eta} Q}\left(\left(m_{\eta}-m_{3}\right)^{2}-s_{3}\right)-1,
$$

where $Q$ is the energy of the reaction, $Q=m_{\eta}-m_{1}-m_{2}-m_{3}$. The square of the invariant amplitude of the considered process is then parametrized using the polynomial

$$
|\mathcal{M}|^{2}=|A|^{2}\left(1+a y+b y^{2}+c x+d x^{2}+e x y+f y^{3}+g x^{2} y+\cdots\right)
$$

with real parameters $a, b, \ldots$.

However, from the unitarity of the theory, we know that amplitudes can possess some nonanalyticities. For instance, the two-pion loop generates a branching point at $4 m_{\pi}^{2}$. For data analyses, usually, only amplitudes within the physical region are relevant. It can happen that this point is sufficiently outside of the physical region, thereby unaffecting the experimental parametrization.

But various intermediate particles can manifest themselves as non-analyticities in the physical region. For example, in the processes with the neutral pion pair in the finite state, there could be a contribution of the charged-pion pair intermediate state and as $m_{\pi^{0}}<m_{\pi^{ \pm}}$, the charged pion branching point occurs in the physical region and it leads to the cusp effect. (Note that its strength is sensitive to $\pi^{+} \pi^{-} \rightarrow \pi^{0} \pi^{0}$ scattering and can be used for an analysis of this process.)

Therefore, polynomial parametrizations cannot describe well the amplitudes with these effects. One has to use more complicated theory-inspired parametrizations (sometimes partially giving up model independence). Nowadays, dominantly those following from the non-relativistic effective field theory [12] or various dispersive appoaches [13] are used.

Our aim has been to construct a fully relativistic parametrization valid at the two-loop level in the low-energy expansion possessing all the analytic properties correctly and taking into account the isospin breaking connected to the $m_{\pi^{0}} \neq m_{\pi^{ \pm}}$difference. The isospin breaking effects stemming 


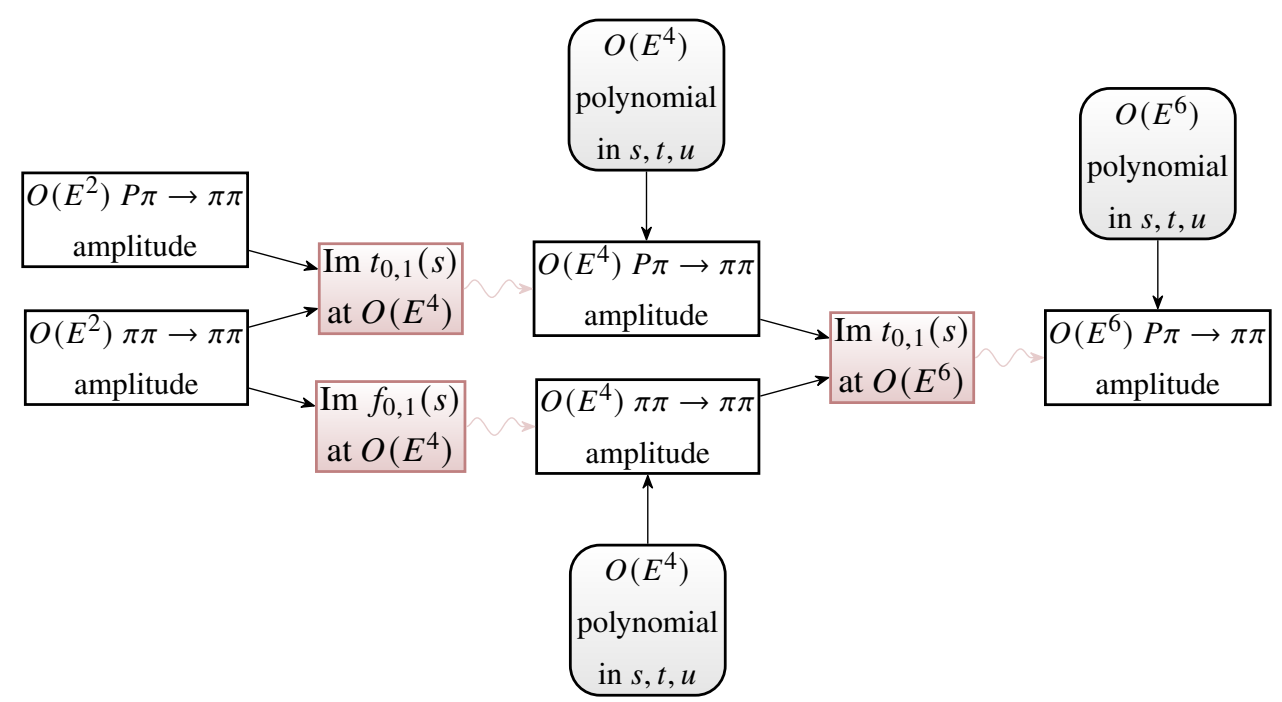

Figure 1: The typical reconstruction procedure of the amplitude for scattering process $P \pi \rightarrow \pi \pi . t_{0,1}(s)$ and $f_{0,1}(s)$ are $\mathrm{S}$ and $\mathrm{P}$ partial waves of $P \pi \rightarrow \pi \pi$ and $\pi \pi \rightarrow \pi \pi$ amplitudes, respectively.

from the electromagnetic interactions (such as the exchange of the virtual photons, photon emission) are neglected.

\section{Construction of the parametrization for a scattering process of stable particles}

We generalize the method of the "reconstruction theorem," first established in [14]. The tools we use for such construction are the following. From the analyticity of the amplitudes (which is connected to the principle of causality), we can deduce the dispersion relations (DRs) that enable a construction of the full amplitude from the knowledge of its analytic structure and the values of the discontinuities along the branch cuts up to a polynomial.

From the unitarity (which is connected to the principle of conservation of the probability), we can obtain the imaginary part of amplitude from the amplitudes of the intermediate processes.

The importance of individual contributions of various parts and amplitudes is given by the assumption of the low energy behavior of the partial waves ( $E$ is the energy scale of the process),

$$
\operatorname{Re} t_{\ell=0,1}=O\left(E^{2}\right), \quad \operatorname{Re} t_{\ell \geq 2}=O\left(E^{4}\right), \quad \operatorname{Im} t_{\ell=0,1}=O\left(E^{4}\right), \quad \operatorname{Im} t_{\ell \geq 2}=O\left(E^{8}\right),
$$

which stems from the chiral counting in the chiral perturbation theory.

The last tool that matches the output of the unitarity relations and the input of DRs is the real analyticity of the amplitude. It tells that the discontinuity along the branch cut of the amplitude is (up to a factor) equal to its imaginary part. (The details for all of the tools are discussed in [1].)

The typical reconstruction procedure of the scattering process proceeds as illustrated in Fig. 1. We take the leading order amplitudes as the input. The unitarity tells us the imaginary part of the amplitude to the higher order in $O(E)$, which is afterward used in the dispersion relations that gives us up to a polynomial the considered amplitude to this higher precision. In a similar way, we can construct the $\pi \pi$ scattering amplitudes, which enables us to perform the second step, thereby obtaining the amplitude to the two-loop level. It is parameterized by coefficients of the polynomials. 


\section{Construction of the parametrization for processes with unstable particles}

The procedure described in the previous section works well for scattering processes of stable particles $[15,16]$. In order to obtain the amplitude of a decay process, one can, in principle, perform an analytic continuation in Mandelstam variables into the decay region. However, there appears a complication for an unstable particle $P$ as the real analyticity is lost and there is no direct connection between the imaginary part and the discontinuity. One way to overcome this obstacle is to construct the amplitudes in a fictitious world, where the mass of particle P is stable, i.e., $M_{P}<3 m_{\pi^{0}}$ and perform afterwards an analytic continuation in $M_{P}$. The main question of such a procedure is how to find the correct analytic continuation that leads to the right physical amplitude.

For that end, we use the fact that for our procedure, it is enough to require the right analytic procedure only in the region where the low-energy expansion works, and there it should be the same as the one obtained by using the Feynman diagram method in some effective theory with the same expansion. Therefore, the study of the analytic properties of all the possible diagrams that could contribute to such a process leads us to the correct continuation in $M_{P}$.

For the various cases, it is the following. The analysis at the one-loop level is simple even if we consider different masses of the pions, $m_{\pi^{0}} \neq m_{\pi^{ \pm}}$. At the two-loop level for $m_{\pi^{0}}=m_{\pi^{ \pm}}$, the analysis was performed already in [17, 18]. In [1], we have succeeded in its extension for the isospin breaking in the decays of the neutral particles that possess the cusp effect, $K_{L} \rightarrow 3 \pi^{0}$ and $\eta \rightarrow 3 \pi^{0}$. The analyses for the remaining processes at the two-loop level remain to be done.

\section{Two-loop parametrization of $K \rightarrow 3 \pi$ and $\eta \rightarrow 3 \pi$ amplitudes}

Using the methods and prescriptions given in the previous sections, we have obtained the wanted parametrizations [1]. As an example, we show here the structure of the one for $\eta \rightarrow 3 \pi^{0}$ decay amplitude for $m_{\pi^{0}} \neq m_{\pi^{ \pm}}$.

The amplitude consists of two parts,

$$
\mathcal{M}_{00}^{\eta}=P_{00}^{\eta}(s, t, u)+U_{00}^{\eta}(s, t, u)+O\left(E^{8}\right),
$$

where the polynomial part

$$
P_{00}^{\eta}(s, t, u)=A_{\eta}+C_{\eta} \frac{\left[\left(s-s_{0}\right)^{2}+\left(t-s_{0}\right)^{2}+\left(u-s_{0}\right)^{2}\right]}{F_{\pi}^{4}}+E_{\eta} \frac{\left[\left(s-s_{0}\right)^{3}+\ldots\right]}{F_{\pi}^{6}}
$$

depends on three parameters $A_{\eta}, C_{\eta}$ and $E_{\eta}$ and the unitary part

$$
U_{00}^{\eta}(s, t, u)=16 \pi\left[\mathcal{W}_{\eta ; 00}(s)+\mathcal{W}_{\eta ; 00}(t)+\mathcal{W}_{\eta ; 00}(u)\right]
$$

as its name suggests follows from the unitarity and within $\mathcal{W}_{\eta ; 00}(s)$, it contains various kinematic functions ${ }^{1} \tilde{K}_{i}, i=1, \ldots, 17$ multiplied by polynomials in $s$ depending (linearly) on the parameters

\footnotetext{
${ }^{1}$ The correct analytic structure is hidden in those kinematic functions $\tilde{K}_{i}$. We have a closed form for some of them, but the majority has to be computed using dispersive integrals

$$
\widetilde{K}_{i}(s)=\frac{s}{\pi} \int_{4 M_{\pi}^{2}}^{\infty} \frac{d x}{x} \frac{\widetilde{k}_{i}(x)}{x-s-\mathrm{i} 0} .
$$


$A_{\eta}, C_{\eta}$ and the other parameters of the one-loop $\eta \rightarrow \pi^{ \pm} \pi^{\mp} \pi^{0}$ and $\pi \pi$ scattering processes. If we take these other parameters as known from some additional analyses, the amplitude merely depends on three parameters. Note, however, that we need a square of the amplitude for a data fit, so there is a quadratic dependence on the parameters.

\section{Conclusions and the outlook}

We have obtained a parametrization of $K \rightarrow 3 \pi$ and $\eta \rightarrow 3 \pi$ decay amplitudes possessing the correct analytic properties at two loops (and all the ingredients in a closed analytic form). For the processes $K_{L} \rightarrow 3 \pi^{0}$ and $\eta \rightarrow 3 \pi^{0}$, it also includes the isospin breaking connected with $m_{\pi^{0}} \neq m_{\pi^{ \pm}}$. Such an extension for the other processes is still in progress.

The connection with the chiral perturbation theory can be easily restored and one can, e.g., study the effects of final state rescattering on its result (as illustrated in [11]). We prepare fits of the data for $\eta \rightarrow 3 \pi^{0}$ (with the cusp) and $\eta \rightarrow \pi^{+} \pi^{-} \pi^{0}[4,5,19,20]$. An interesting extension of the analysis to the process $\eta^{\prime} \rightarrow \eta \pi \pi$ can be possibly obtained by addition of the $1 / N_{C}$ expansion [21, 22], as this process was recently studied in high-precision experiments [23, 24].

\section{Acknowledgments}

This work is supported in part by grant N. 40652ZE, financed by the French Ministry of Foreign Affairs and the French Ministry for Higher Education, Research and Innovation, and by grant N. 7AMB18FR039, financed by the Czech Ministry for Education, Youth and Sports, both within the framework of the bilateral PHC Barrande Mobility Program 2018, and by the Czech Science Foundation Project N. GACR 18-17224S.

\section{References}

[1] K. Kampf, M. Knecht, J. Novotný and M. Zdráhal, Dispersive construction of two-loop $P \rightarrow \pi \pi \pi(P=K, \eta)$ amplitudes, Physical Review D 101 (2020) 074043 [1911.11762].

[2] NA48/2 collaboration, Empirical parameterization of the $K^{ \pm} \rightarrow \pi^{ \pm} \pi^{0} \pi^{0}$ decay Dalitz plot, Physics Letters B 686 (2010) 101 [1004 . 1005].

[3] Crystal Ball at MAMI collaboration, Determination of the Dalitz plot parameter $\alpha$ for the decay $\eta \rightarrow 3 \pi^{0}$ with the Crystal Ball@MAMI-B, Eur. Phys. J. A 39 (2009) 169 [0812. 3324].

[4] KLOE-2 collaboration, Precision measurement of the $\eta \rightarrow \pi^{+} \pi^{-} \pi^{0}$ Dalitz plot distribution with the KLOE detector, JHEP 05 (2016) 019 [1601.06985].

[5] A2 collaboration, High-statistics measurement of the $\eta \rightarrow 3 \pi^{0}$ decay at the Mainz Microtron, Physical Review C 97 (2018) 065203 [1803.02502].

[6] H.-B. Li, $\eta$ and $\eta^{\prime}$ physics at BES-III, Journal of Physics G 36 (2009) 085009 [0902 . 3032].

[7] L. Gan, Probes for fundamental QCD symmetries and a dark gauge boson via light meson decays, PoS CD15 (2015) 017. 
[8] M. Zdráhal, Determination of the $m_{u}$ and $m_{d}$ quark masses from $\eta \rightarrow 3 \pi$ decay, Nuclear Physics B - Proceedings Supplements 219-220 (2011) 68 [1109. 1835].

[9] L. Gan, B. Kubis, E. Passemar and S. Tulin, Precision tests of fundamental physics with $\eta$ and $\eta^{\prime}$ mesons, 2007.00664.

[10] N. Cabibbo, Determination of the $a_{0}-a_{2}$ pion scattering length from $K^{+} \rightarrow \pi^{+} \pi^{0} \pi^{0}$ decay, Physical Review Letters 93 (2004) 121801 [hep-ph/0405001].

[11] K. Kampf, M. Knecht, J. Novotný and M. Zdráhal, Analytical dispersive construction of $\eta \rightarrow 3 \pi$ amplitude: first order in isospin breaking, Physical Review D 84 (2011) 114015 [1103.0982].

[12] J. Gasser, B. Kubis and A. Rusetsky, Cusps in $K \rightarrow 3 \pi$ decays: a theoretical framework, Nuclear Physics B 850 (2011) 96 [1103 .4273].

[13] G. Colangelo, S. Lanz, H. Leutwyler and E. Passemar, Dispersive analysis of $\eta \rightarrow 3 \pi$, The European Physical Journal C 78 (2018) 947 [1807.11937].

[14] J. Stern, H. Sazdjian and N.H. Fuchs, What $\pi-\pi$ scattering tells us about chiral perturbation theory, Physical Review D 47 (1993) 3814 [hep-ph/9301244].

[15] M. Knecht, B. Moussallam, J. Stern and N.H. Fuchs, The low-energy $\pi \pi$ amplitude to one and two loops, Nuclear Physics B 457 (1995) 513 [hep-ph/9507319].

[16] M. Zdráhal and J. Novotný, Dispersive approach to chiral perturbation theory, Physical Review D 78 (2008) 116016 [http: //arxiv.org/abs/0806.4529v2].

[17] C. Kacser, Analytic structure of partial-wave amplitudes for production and decay processes, Physical Review 132 (1963) 2712.

[18] J.B. Bronzan and C. Kacser, The Khuri-Treiman representation and perturbation theory, Physical Review 132 (1963) 2703.

[19] WASA-Aт-COSY collaboration, Measurement of the $\eta \rightarrow \pi^{+} \pi^{-} \pi^{0}$ Dalitz plot distribution, Physical Review C 90 (2014) 045207 [1406.2505].

[20] BESIII collaboration, Measurement of the matrix elements for the decays $\eta \rightarrow \pi^{+} \pi^{-} \pi^{0}$ and $\eta / \eta^{\prime} \rightarrow \pi^{0} \pi^{0} \pi^{0}$, Physical Review D 92 (2015) 012014 [1506.05360].

[21] P. Herrera-Siklody, J.I. Latorre, P. Pascual and J. Taron, Chiral effective lagrangian in the large N(c) limit: The nonet case, Nuclear Physics B 497 (1997) 345 [hep-ph/9610549].

[22] R. Kaiser and H. Leutwyler, Large N(c) in chiral perturbation theory, The European Physical Journal C 17 (2000) 623 [hep-ph/0007101].

[23] P. Adlarson et al., Measurement of the decay $\eta^{\prime} \rightarrow \pi^{0} \pi^{0} \eta$ at MAMI, Physical Review D 98 (2018) 012001 [1709.04230].

[24] BESIII collaboration, Measurement of the matrix elements for the decays $\eta^{\prime} \rightarrow \eta \pi^{+} \pi^{-}$and $\eta^{\prime} \rightarrow \eta \pi^{0} \pi^{0}$, Physical Review D 97 (2018) 012003 [1709.04627]. 